\title{
Mucinous cystadenoma of a horseshoe kidney: A case report and literature review
}

\author{
Taku Mitome, MD; ${ }^{*}$ Masahiro Yao, MD; ${ }^{1}$ Naoko Udaka, MD;" Shusei Fusayasu, MD;" Koji Izumi, MD;* \\ Kimito Osaka, MD; ${ }^{*}$ Narihiko Hayashi, MD; ${ }^{*}$ Noboru Nakaigawa, MD; ${ }^{*}$ Yoji Nagashima, MD; ${ }^{+}$ \\ Yoshinobu Kubota, $M D^{*}$
}

*Department of Urology, Yokohama City University Graduate School of Medicine, Yokohama, Kanagawa, Japan; ‘Department of Molecular Pathology, Yokohama City University Graduate School of Medicine, Yokohama, Kanagawa, Japan; §Department of Pathology, Yokohama City University Hospital, Yokohama, Kanagawa, Japan

Cite as: Can Urol Assoc J 2015;9(1-2):E30-2. http://dx.doi.org/10.5489/cuaj.2211

Published online January 12, 2015.

\section{Abstract}

A 45-year-old man complained of a palpable mass in his left abdomen. Computed tomography showed a horseshoe kidney with a Bosniak type II complicated cyst from a left segment. Three years after his initial examination, due to the growing cystic lesion and the compression imposed on the urinary collecting system and surrounding organs, we performed a left heminephrectomy. The diagnosis was mucinous cystadenoma of the kidney. No recurrence was observed 6 months after surgery. The histopathology was unique since the inner surface of the cyst was covered by a mucin-positive columnar epithelium connected to a urothelium, with continuous transition between the two. This suggests that the mucinous tumour may have originated from a sequestered segment of the renal pelvic epithelium in the renal parenchyma.

\section{Introduction}

Primary mucinous cystadenoma occurring in a horseshoe kidney is extremely rare, with only 2 reported cases in the English literature. ${ }^{1-3}$ We present a third case, which mimicked a Bosniak type IIF renal cyst.

\section{Case report}

A 45-year-old man visited our clinic with a palpable mass in the left abdomen. Computed tomography (CT) showed a horseshoe kidney and a complicated cyst categorized as Bosniak type IIF, with a maximum diameter of $80 \mathrm{~mm}$ arising from a left segment of the kidney (Fig. 1, part A). The density of the cyst content on plain $\mathrm{CT}$ ranged from 10 to 20 Hounsfield units (HU). Dynamic CT scanning showed a thin wall with slight contrast, but there was no contrast of the cyst content. Magnetic resonance imaging (MRI) yield- ed a low-intensity T1-weighted image and a high-intensity T2-weighted image, and no signs suggesting malignancy. The blood and urine tests were within the normal range. Because the patient preferred active surveillance rather than surgical intervention, we followed up at intervals of 6 months by CT. Three years after his initial examination, the cystic lesion had asymptomatically grown to $165 \mathrm{~mm}$ in diameter, and hydronephrosis of the left renal pelvis appeared (Fig. 1, part B). We therefore performed an ultrasonography-guided percutaneous cyst aspiration. Unexpectedly, however, only a small amount of mucinous liquid with high viscosity was aspirated. Cytological and microbiological examinations of the aspirated material revealed no neoplastic cells and no bacteria, respectively.

A cyst excision was planned due to the compression imposed on the patient's urinary collecting system and surrounding organs. During the excision, we observed that the content of the cyst included a significant amount of mucin with a few blood clots and tissue fragments. Therefore, we performed a left heminephrectomy (Fig. 2). The total weight of the cyst content was $1.5 \mathrm{~kg}$, and the cyst was $150 \times 100 \times 100 \mathrm{~mm}$. The inner surface of the cyst wall was macroscopically smooth, and there was no tumorous protraction or transportation to the collecting system. Histopathologic examinations demonstrated an inner surface lined with a single layer of urothelium, a single layer of columnar epithelium, or both, with continuous transition between them (Fig. 3, part A). There were mucin-positive cells in the columnar epithelium, and the columnar epithelium protruded into the inner mucin pools (Fig. 3, part B). The final diagnosis was mucinous cystadenoma of the kidney. No recurrence was observed 6 months after surgery.

\section{Discussion}

We report an extremely rare case of mucinous cystadenoma in a horseshoe kidney. To our knowledge, there have been 


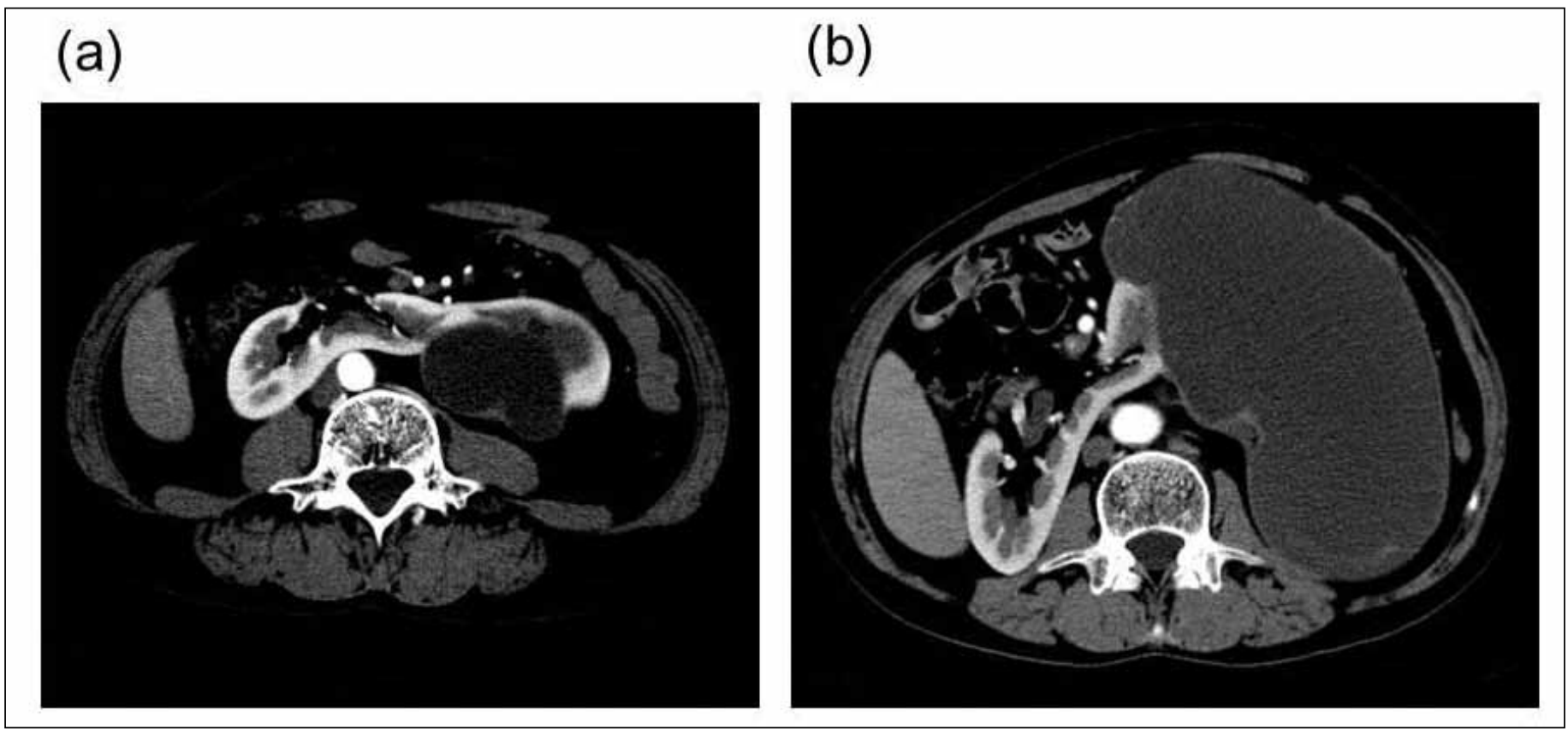

Fig. 1. A: A computed tomography at the initial onset showing a complicated cyst with a maximum diameter of $80 \mathrm{~mm}$ arising from a left segment of the horseshoe kidney. B: Three years later, the cystic lesion grew to $165 \mathrm{~mm}$ in diameter.

only 12 reported cases of mucinous cystadenoma of the kidney, and only 2 of such cases occurred in a horseshoe kidney, both of which were reported in the English literature.

Among these 13 cases, included the present case, 8 $(61.5 \%)$ occurred in men and 5 in women. ${ }^{1-9}$ The median age was 59 (range: 27-79). The median size of the cystic tumour was $156 \mathrm{~mm}$ (range: $35-370) .^{1-9}$ Despite their large size, 6 tumours (46.1\%) were asymptomatic and detected incidentally by ultrasonography and/or CT., ${ }^{1,2,5}$ Seven patients $(53.8 \%)$ had symptoms related to pyelonephritis or local pain. $3,4,6,8,9$

It can be quite difficult to distinguish a mucinous cystadenoma from a renal cyst based on physical findings, laboratory data, and imaging studies, including ultrasonography, $\mathrm{CT}$, and MRI. None of the previous reports correctly diagnosed the disease before surgery; the final diagnosis was made only after tumour excision. The density of simple renal cysts ranges from -10 to $+20 \mathrm{HU}$ on $\mathrm{CT} .{ }^{2}$ With our patient, the density of the cystadenoma ranged from 10 to $20 \mathrm{HU}$ and the radiologic features mimicked a renal parenchymal cyst. $^{2}$ Mucinous cystadenoma of the kidney may therefore be underdiagnosed by routine imaging studies.

In the previous reports, the cystadenoma originated from the urinary collecting system in 8 cases and from the renal parenchyma in the remaining 4 , based on histological findings. ${ }^{1-9}$ Some authors have postulated that the development of mucinous cystadenoma with an intestinal phenotype in the kidney involves urolithiasis, infections, or both, and these events may induce intestinal metaplasia. ${ }^{6}$ In fact, urolithiasis was reported in 7 cases..$^{1,5-9}$ In contrast, Liwnicz and colleagues suggested that the formation of stones may be secondary to the mucin secretion from the cystic tumour. ${ }^{10}$ They proposed that the secreted mucin may have caused neoplastic formations after fusing with sodium and calcium. On the other hand, other authors have speculated that the mucinous tumours may originate from a sequestered segment of the renal pelvic epithelium in the renal parenchyma, especially in the case of an anomalous kidney, such as a horseshoe kidney. ${ }^{2,3}$ The present case was also associated with horseshoe kidney, and the patient did not have a history of urolithiasis or infections. In mucinous cystadenomas

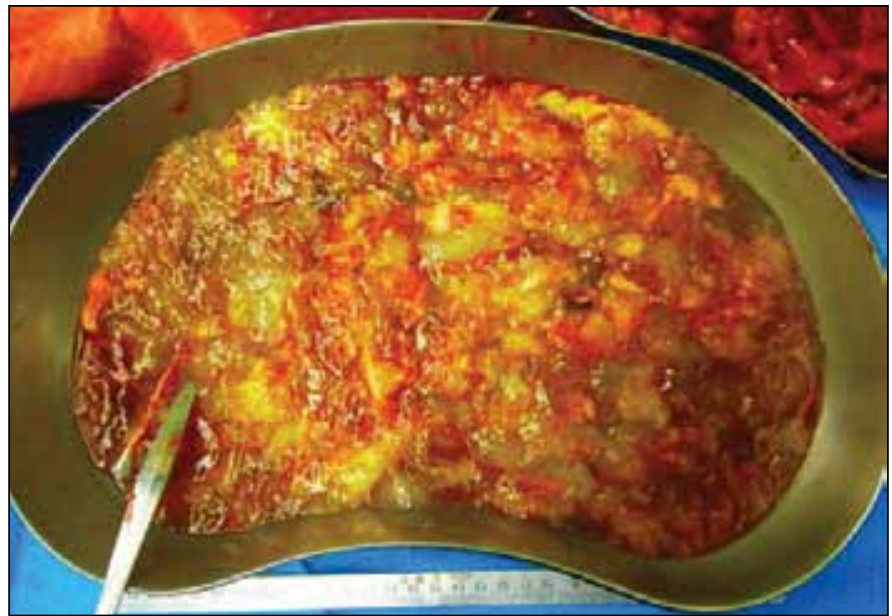

Fig. 2. Macroscopic findings of the cyst removed during surgery. The content included a large amount of mucin with a few blood clots and tissue fragments. The total weight of the contents was 1.5 kilograms. 
(a)

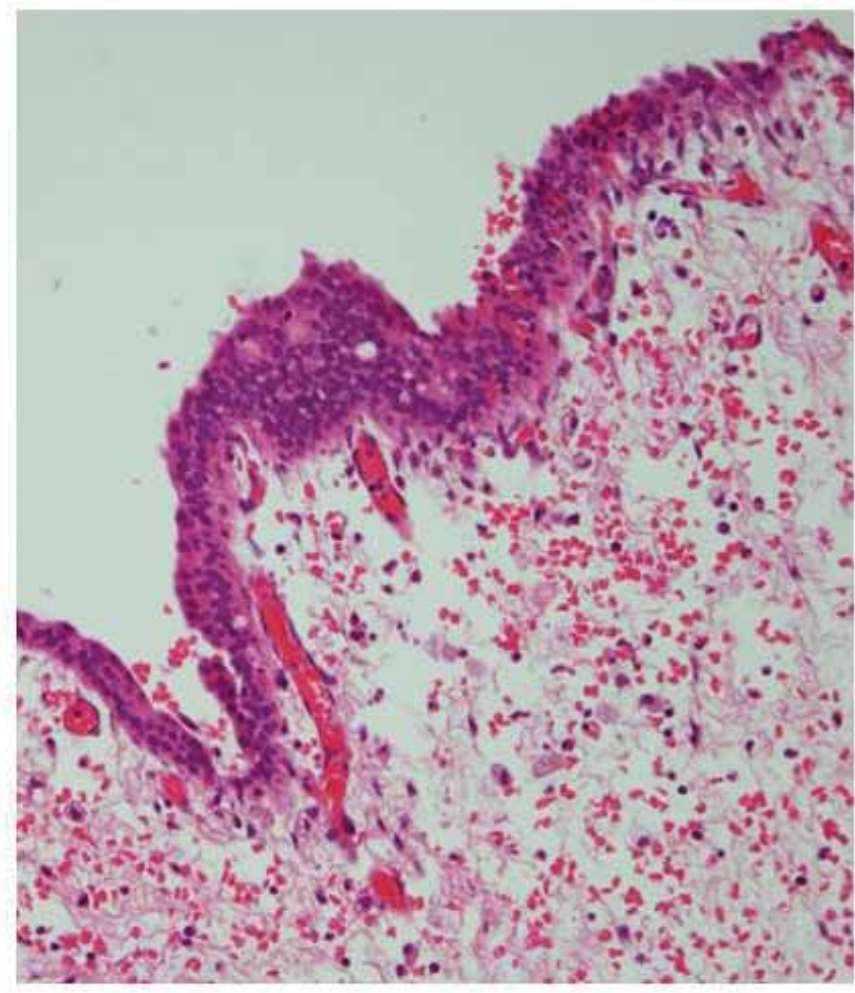

(b)

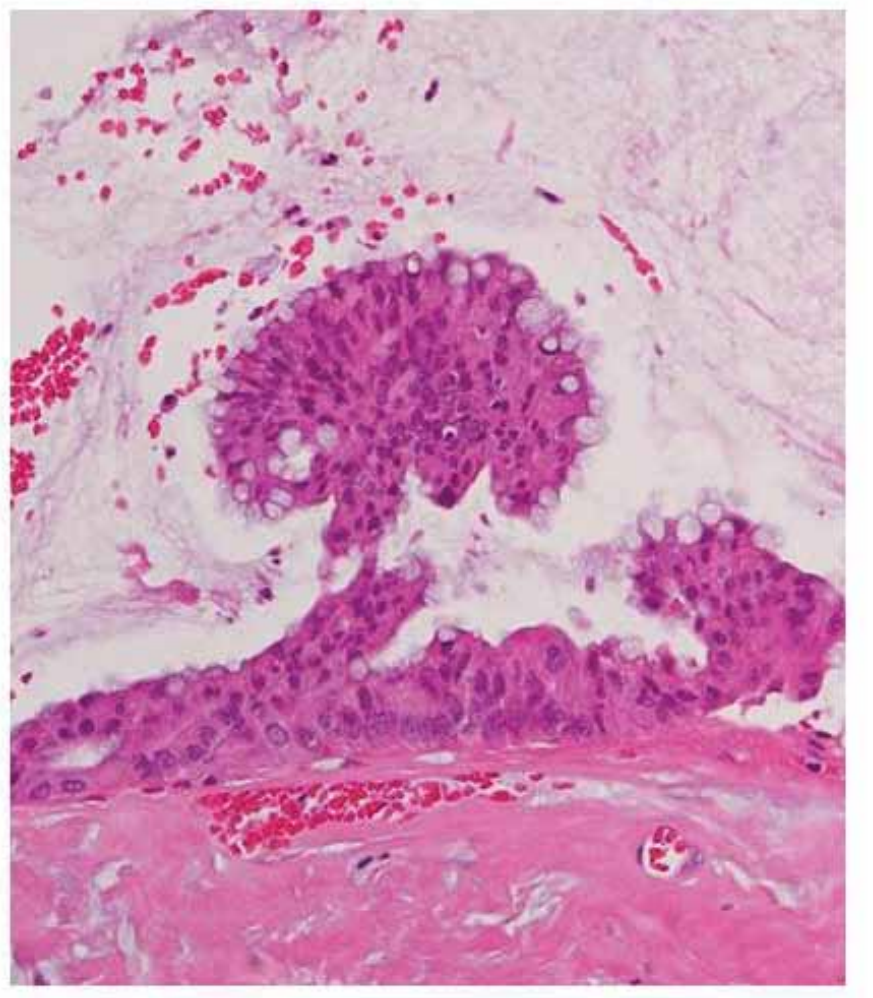

Fig. 3. Histopathologic examination of the cystadenoma. A: The cyst wall was fibrous, and there was an area of urothelium on the inner surface of the cyst

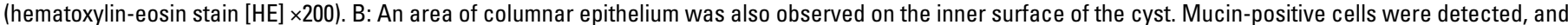
the columnar epithelium protruded into the inner mucin pools $(\mathrm{HE} \times 200)$.

arising from the renal pelvis, the inner surface of the cyst is usually covered by urothelium. ${ }^{7}$ In those arising from the renal parenchyma, which include the 2 cases reported in horseshoe kidneys, the inner surface was not covered by urothelium. In our case, the histopathologic findings demonstrated an inner surface of the cyst covered by a mucinpositive columnar epithelium connected to urothelium, with continuous transition between the two, suggesting that the mucinous tumour may have originated from a sequestered segment of the renal pelvic epithelium in the renal parenchyma.

\section{Conclusion}

We report an extremely rare case of mucinous cystadenoma of a horseshoe kidney. The details of the case suggest that the mucinous tumour may have originated from a sequestered segment of the renal pelvic epithelium in the renal parenchyma.

Competing interests: Authors declare no competing financial or personal interests.

This paper has been peer-reviewed.

\section{References}

1. Chablé-Montero F, Mendoza-Ramírez S, Lavenant-Borja M, et al. Mucinous cystadenoma of the pyelocaliceal system: A report of 3 examples and an analysis of 17 previously published cases. Ann Diagn Pathol 2013;17:239-44. http://dx.doi.org/10.1016/i.anndiagpath.2012.10.008

2. Akan $\mathrm{H}$, Dalva I, Yildiz 0 , et al. Mucinous cystadenoma mimicking simple renal parenchymal cyst in a horseshoe kidney. Int J Urol 2005;12:493-6. http://dx.doi.org/10.1111/j.1442-2042.2005.01078.x

3. Ross DG, D'Amato NA. Papillary mucinous cystadenoma of probable renal pelvic origin in a horseshoe kidney. Arch Pathol Lab Med 1985;109:954-5.

4. Charfi S, Ayadi L, Khabir A, et al. Mucinous cystadenoma of the kidney. Prog Urol 2008;18:613-6. http://dx.doi.org/10.1016/i.purol.2008.03.012

5. Kumar B, Agarwal RK, Upadhyay P. Mucinous cystadenoma of the kidney. Indian J Pathol Microbiol 2009;52:274-5. http://dx.doi.org/10.4103/0377-4929.48945

6. Arakawa $M$, Jimi $A$, Ootomi $M$, et al. A mucin-producing cystadenoma, borderline malignancy, of the renal pelvis and ureter: A case report [in Japanese]. Gan No Rinsho 1989;35:499-504.

7. Gangane N, Anshu, Shende N, et al. Mucinous cystadenoma arising from renal pelvis: A report of 2 cases. Urol J 2008;5:197-9.

8. Ohyabu Y, Sameshima H, Eto K. Mucin-producing cystadenoma (borderline malignancy) of the renal pelvis and ureter. A case report [in Japanese]. Nihon Hinyokika Gakkai Zasshi 1990;81:913-6. http://dx.doi. org/10.5980/ipniurol1989.81.913

9. Park S, Meng MV, Greenberg MS, et al. Muconephrosis. Urology 2002;60:344. http://dx.doi. org/10.1016/S0090-4295(02)01707-7

10. Liwnicz BH, Lepow H, Schutte H, et al. Mucinous adenocarcinoma of the renal pelvis: Discussion of possible pathogenesis. J Urol 1975;114:306-10.

Correspondence: Dr. Taku Mitome, Department of Urology, Yokohama City University Graduate School of Medicine, 3-9 Fukuura, Kanazawa-ku, Yokohama, Kanagawa, 236-0004 Japan; e053053a@yahoo.co.jp 